REPRESENTATION THEORY

An Electronic Journal of the American Mathematical Society

Volume 14, Pages 264-284 (March 8, 2010)

S 1088-4165(10)00370-5

\title{
THE SATAKE ISOMORPHISM FOR SPECIAL MAXIMAL PARAHORIC HECKE ALGEBRAS
}

\author{
THOMAS J. HAINES AND SEAN ROSTAMI
}

\begin{abstract}
Let $G$ denote a connected reductive group over a nonarchimedean local field $F$. Let $K$ denote a special maximal parahoric subgroup of $G(F)$. We establish a Satake isomorphism for the Hecke algebra $\mathcal{H}_{K}$ of $K$-bi-invariant compactly supported functions on $G(F)$. The key ingredient is a Cartan decomposition describing the double coset space $K \backslash G(F) / K$. As an application we define a transfer homomorphism $t: \mathcal{H}_{K^{*}}\left(G^{*}\right) \rightarrow \mathcal{H}_{K}(G)$ where $G^{*}$ is the quasi-split inner form of $G$. We also describe how our results relate to the treatment of Cartier [Car], where $K$ is replaced by a special maximal compact open subgroup $\widetilde{K} \subset G(F)$ and where a Satake isomorphism is established for the Hecke algebra $\mathcal{H}_{\widetilde{K}}$.
\end{abstract}

\section{INTRODUCTION}

The Satake isomorphism plays an important role in automorphic forms and in representation theory of $p$-adic groups. For global applications, one may often work with unramified groups. We begin by recalling the Satake isomorphism in this context. Let $G$ denote an unramified group over a nonarchimedean local field $F$. Let $v_{F}$ denote a special vertex in the Bruhat-Tits building $\mathcal{B}\left(G_{\text {ad }}(F)\right)$. Let $\widetilde{K}=\widetilde{K}_{v_{F}}$ denote a special maximal compact open subgroup of $G(F)$ which fixes $v_{F}$. Let

$$
\mathcal{H}_{\widetilde{K}}=C_{c}^{\infty}(\widetilde{K} \backslash G(F) / \widetilde{K})
$$

denote the Hecke algebra of $\widetilde{K}$-bi-invariant compactly-supported complex-valued functions on $G(F)$. Let $A$ denote a maximal $F$-split torus in $G$ whose corresponding apartment in $\mathcal{B}\left(G_{\text {ad }}(F)\right)$ contains $v_{F}$. Let $W=W(G, A)$ denote the relative Weyl group. Then the Satake isomorphism is a $\mathbb{C}$-algebra isomorphism

$$
\mathcal{H}_{\widetilde{K}} \widetilde{C}\left[X_{*}(A)\right]^{W} .
$$

(See [Car.). A key ingredient is the Cartan decomposition

$$
\widetilde{K} \backslash G(F) / \widetilde{K} \cong W(G, A) \backslash X_{*}(A) .
$$

Now let $G$ denote an arbitrary connected reductive group over $F$ and let $\widetilde{K}, v_{F}$ and so on have the same meaning as above. A form of the Satake isomorphism for

Received by the editors October 17, 2009 and, in revised form, November 29, 2009.

2010 Mathematics Subject Classification. Primary 11E95, 20G25; Secondary 22E20.

The first author was partially supported by NSF Focused Research Grant DMS-0554254 and NSF Grant DMS-0901723, and by a University of Maryland GRB Semester Award.

(C)2010 American Mathematical Society Reverts to public domain 28 years from publication 
such $G$ was described by Cartier Car, but it is less explicit than that above. It identifies $\mathcal{H}_{\widetilde{K}}$ with the ring of functions

$$
\mathbb{C}\left[M(F) / M(F)^{1}\right]^{W},
$$

where $M:=\operatorname{Cent}_{G}(A)$ is a minimal $F$-Levi subgroup of $G$ and $M(F)^{1}$ is the unique maximal compact open subgroup of $M(F)$. The quotient $M(F) / M(F)^{1}$ is a free abelian group $\widetilde{\Lambda}_{M}$ which contains $X_{*}(A)$ and has the same rank. (In [Car], our $\widetilde{\Lambda}_{M}$ is denoted $\Lambda(M)$ or simply $\Lambda$.) As Cartier explains, in this general context we have a Satake isomorphism

$$
\mathcal{H}_{\widetilde{K}} \cong \mathbb{C}\left[\widetilde{\Lambda}_{M}\right]^{W},
$$

and a Cartan decomposition

$$
\widetilde{K} \backslash G(F) / \widetilde{K} \cong W(G, A) \backslash \widetilde{\Lambda}_{M} .
$$

However, Cartier does not identify $\widetilde{\Lambda}_{M}$ explicitly, except in special cases.

Now let $K=K_{v_{F}}$ denote the special maximal parahoric subgroup of $G(F)$ corresponding to $v_{F}$; it is a normal subgroup of $\widetilde{K}_{v_{F}}$ having finite index (see Section 8). This paper concerns the Hecke algebra $\mathcal{H}_{K}=C_{c}^{\infty}(K \backslash G(F) / K)$. In several situations, it is more appropriate to consider $\mathcal{H}_{K}$ instead of $\mathcal{H}_{\widetilde{K}}$, for example, in relation to Shimura varieties having parahoric level structure (see Rap and [H05]).

Let $M(F)_{1} \subset M(F)$ denote the unique parahoric subgroup of $M(F)$; it is a finite-index normal subgroup of $M(F)^{1}$. Our main result is the following theorem.

Theorem 1.0.1. Let $\Lambda_{M}:=M(F) / M(F)_{1}$. There is a canonical isomorphism

$$
\mathcal{H}_{K} \underset{\rightarrow}{ } \mathbb{C}\left[\Lambda_{M}\right]^{W} \text {. }
$$

The group $\Lambda_{M}$ is a finitely generated abelian group which can be explicitly described and which has the property that $\widetilde{\Lambda}_{M}=\Lambda_{M} /$ tor sion. Moreover, $\widetilde{K} / K \cong \Lambda_{M \text {,tor }}$, the torsion subgroup of $\Lambda_{M}$.

When $G$ is unramified over $F$ or when $G$ is semi-simple and simply connected, it turns out that $\widetilde{K}=K$ and $\widetilde{\Lambda}_{M} \cong \Lambda_{M}$ (see Section[11) so that our theorem does not give any new information in those cases. However, our results are new in the case $\widetilde{K} \neq K$, and different methods from $\widetilde{C a r}$ are needed to prove them. For ramified groups, in particular, our results are expected to play some role in the study of Shimura varieties with parahoric level structure at $p$. For more about ramified groups and Shimura varieties with parahoric level the reader should consult Rap, $[\mathrm{PR}$, and $[\mathrm{Kr}]$.

In order to describe $\Lambda_{M}$, we need to recall some notation and results of Kottwitz Ko97. Let $F^{s}$ denote a separable closure of $F$, and let $F^{\text {un }}$ denote the maximal unramified extension of $F$ in $F^{s}$. Let $L=\widehat{F^{u n}}$ denote the completion of $F^{\text {un }}$ with respect to the valuation on $F^{\text {un }}$ which extends the normalized valuation on $F$. Let $I=\operatorname{Gal}\left(F^{s} / F^{\text {un }}\right) \cong \operatorname{Gal}\left(L^{s} / L\right)$ denote the inertia subgroup of $\operatorname{Gal}\left(F^{s} / F\right)$, and let $\sigma \in \operatorname{Aut}(L / F)$ denote the Frobenius automorphism. In Ko97] Kottwitz defined a surjective homomorphism

$$
\kappa_{G}: G(L) \rightarrow X^{*}(Z(\widehat{G}))_{I},
$$

and in loc. cit. $§ 7.7$ he also proved that this induces a surjective homomorphism

$$
\kappa_{G}: G(F) \rightarrow X^{*}(Z(\widehat{G}))_{I}^{\sigma}
$$


of the groups of $\sigma$-invariants. Set $G(L)_{1}:=\operatorname{ker}\left(\kappa_{G}\right)$ and $G(F)_{1}:=G(F) \cap G(L)_{1}$. (When $G=M$, this is consistent with our definition of $M(F)_{1}$ above; see Lemmas 4.1.1, 4.2.1)

The Iwahori-Weyl group $\widetilde{W}$ (defined in $₫ 2.3$ ) for $G$ carries a natural action under $\sigma$ and contains a $\sigma$-invariant abelian subgroup $\Omega_{G}$ (the subgroup of length-zero elements). By choosing representatives in the normalizer of $A$ we may embed $\widetilde{W}^{\sigma}$ set-theoretically into $G(F)$, and then $\Omega_{G}^{\sigma}$ is mapped by $\kappa_{G}$ isomorphically onto $X^{*}(Z(\widehat{G}))_{I}^{\sigma}$ (see Section 2). The following is the sought-after explicit description of $\Lambda_{M}$ :

Proposition 1.0.2. The Kottwitz homomorphism induces an isomorphism

$$
\Lambda_{M}=M(F) / M(F)_{1} \cong X^{*}(Z(\widehat{M}))_{I}^{\sigma} .
$$

Via the Kottwitz isomorphism $\kappa_{M}: \Omega_{M}^{\sigma} \underset{\rightarrow}{\rightarrow} X^{*}(Z(\widehat{M}))_{I}^{\sigma}$, we can also identify $\Lambda_{M}$ with $\Omega_{M}^{\sigma}$.

As before, the main step in the proof of Theorem 1.0.1 is an appropriate Cartan decomposition.

Theorem 1.0.3. The embedding $\Omega_{M}^{\sigma} \subset \widetilde{W}^{\sigma} \hookrightarrow G(F)$ determines a bijection

$$
W(G, A) \backslash \Omega_{M}^{\sigma} \cong K \backslash G(F) / K .
$$

Equivalently, via the isomorphism $\kappa_{M}: \Omega_{M}^{\sigma} \widetilde{\rightarrow} X^{*}(Z(\widehat{M}))_{I}^{\sigma}$, we have a bijection

$$
W(G, A) \backslash X^{*}(Z(\widehat{M}))_{I}^{\sigma} \stackrel{\Im}{\rightarrow} K \backslash G(F) / K .
$$

We give additional information about the finitely generated abelian group $\Lambda_{M}$ in Section 11. For example, we prove that if $G$ is an inner form of a split group, then $\Lambda_{M}=X^{*}(Z(\widehat{M}))=X_{*}(T)_{\sigma}$ (see Corollary 11.3.2).

Finally, let $G^{*}$ denote the quasi-split inner form of $G$, and consider special maximal parahoric subgroups $K^{*} \subset G^{*}(F)$ and $K \subset G(F)$. In Section 12, we define a canonical transfer homomorphism $t: \mathcal{H}_{K^{*}}\left(G^{*}\right) \rightarrow \mathcal{H}_{K}(G)$, and we establish some of its basic properties.

This article relies heavily on the ideas of Kottwitz, especially as they are manifested in the article $[\mathrm{HR}$. The main theorems of [HR] provide the starting points for the proof of Theorem 1.0 .3

We thank the referee for some quite helpful suggestions.

\section{Notation}

2.1. Ring-theoretic notation. Let $\mathcal{O}=\mathcal{O}_{F}$ (resp. $\mathcal{O}_{L}$ ) denote the ring of integers in the field $F$ (resp. $L$ ). Let $\varpi$ denote a uniformizer of $F$ (resp. $L$ ), and let $k_{F}$ denote the residue field of $F$. We may identify the residue field $k_{L}$ with an algebraic closure of $k_{F}$. Let $\Gamma:=\operatorname{Gal}\left(F^{s} / F\right)$.

Throughout this paper, if $J \subset G(F)$ denotes a compact open subgroup, we make

$$
\mathcal{H}_{J}:=C_{c}^{\infty}(J \backslash G(F) / J)
$$

a convolution algebra by using the Haar measure on $G(F)$ which gives $J$ volume 1 . 
2.2. Buildings notation. Let $\mathcal{B}(G(L))$ (resp. $\mathcal{B}(G(F))$ ) denote the Bruhat-Tits building of $G(L)$ (resp. $G(F)$ ). The building $\mathcal{B}(G(L))$ carries an action of $\sigma$. By BT2, 5.1.25, we have an identification $\mathcal{B}(G(F))=\mathcal{B}(G(L))^{\sigma}$. Moreover, there is a bijection $\mathbf{a}_{J} \mapsto \mathbf{a}_{J}^{\sigma}$ from the set of $\sigma$-stable facets in $\mathcal{B}(G(L))$ to facets in $\mathcal{B}(G(F))$ (BT2, 5.1.28). This bijection sends alcoves to alcoves (BT2, 5.1.14). It also follows from loc. cit. that every $\sigma$-stable facet $\mathbf{a}_{J}$ in $\mathcal{B}(G(L))$ is contained in the closure $\overline{\mathbf{a}}$ of a $\sigma$-stable alcove $\mathbf{a}$.

Let $v_{F}$ denote a special vertex in $\mathcal{B}\left(G_{\text {ad }}(F)\right)$ (Tits, 1.9$)$. Let $A$ denote a maximal $F$-split torus in $G$ whose corresponding apartment in $\mathcal{B}\left(G_{\text {ad }}(F)\right)$ contains $v_{F}$. Let $\mathcal{A}$ (resp. $\mathcal{A}_{\text {ad }}$ ) denote the apartment in $\mathcal{B}(G(F))$ (resp. $\mathcal{B}\left(G_{\text {ad }}(F)\right)$ ) corresponding to $A$. Let $V_{G(F)}$ denote the real vector space $X_{*}(Z(G))_{\Gamma} \otimes \mathbb{R}$. There exists a simplicial isomorphism (Tits, 1.2 )

$$
\mathcal{A} \cong \mathcal{A}_{\mathrm{ad}} \times V_{G(F)} .
$$

Therefore, there is a minimal dimensional facet $\mathbf{a}_{0}^{\sigma}$ in $\mathcal{A}$ associated to a $\sigma$-stable facet $\mathbf{a}_{0} \subset \mathcal{B}(G(L))$, such that

$$
\mathbf{a}_{0}^{\sigma} \cong\left\{v_{F}\right\} \times V_{G(F)} .
$$

We consider parahoric (or Iwahori) subgroups in the sense of [BT2, 5.2. That is, to a facet $\mathbf{a}_{J} \subset \mathcal{B}(G(L))$ we associate an $\mathcal{O}_{L^{-}}$group scheme $\mathcal{G}_{\mathbf{a}_{J}}^{\circ}$ with connected geometric fibers, whose group of $\mathcal{O}_{L}$-points fixes identically the points of $\mathbf{a}_{J}$. We often write $J(L):=\mathcal{G}_{\mathbf{a}_{J}}^{\circ}\left(\mathcal{O}_{L}\right)$. By [BT2], 5.2, if $\mathbf{a}_{J}$ is $\sigma$-stable, we get a parahoric subgroup $J(F):=J(L)^{\sigma}$ in $G(F)$ and this is associated to the facet $\mathbf{a}_{J}^{\sigma}$ in $\mathcal{B}(G(F))$. Moreover, every parahoric subgroup of $G(F)$ is of this form for a unique $\sigma$-stable facet $\mathbf{a}_{J}$.

Now fix a $\sigma$-stable alcove a whose closure contains $\mathbf{a}_{0}$. Let $I(L)($ resp. $K(L))$ denote the Iwahori (resp. parahoric) subgroup of $G(L)$ corresponding to the $\sigma$ stable alcove a (resp. facet $\mathbf{a}_{0}$ ). Then $I:=I(F)=I(L)^{\sigma}$ is the Iwahori subgroup of $G(F)$ corresponding to $\mathbf{a}^{\sigma}$. Also, $K:=K(F)=K(L)^{\sigma}$ is a special maximal parahoric subgroup of $G(F)$ corresponding to $\mathbf{a}_{0}^{\sigma}$ (or equivalently, to $v_{F}$ ).

2.3. Weyl groups and Iwahori-Weyl groups. For a torus $S$ in $G$, let $N_{G}(S)=$ $\operatorname{Norm}_{G}(S)$ denote its normalizer and $C_{G}(S)=\operatorname{Cent}_{G}(S)$ its centralizer. Let $W(G, S):=N_{G}(S) / C_{G}(S)$ denote its Weyl group.

Fix the torus $A$ as before. From now on, let $S$ be a maximal $L$-split torus that is defined over $F$ and contains $A$ ([BT2], 5.1.12). Let $T:=C_{G}(S)$, a maximal torus of $G$ (defined over $F$ ) since $G_{L}$ is quasi-split by Steinberg's theorem.

We need to recall definitions and facts about Iwahori-Weyl groups; we refer the reader to [HR] for details. Let $T(L)_{1}:=\operatorname{ker}\left(\kappa_{T}\right)$, a normal subgroup of $N_{G}(S)(L)$. Let $\widetilde{W}:=N_{G}(S)(L) / T(L)_{1}$ denote the Iwahori-Weyl group for $G$. It carries an obvious action of $\sigma$. Let $\mathcal{A}_{L}$ denote the apartment of $\mathcal{B}(G(L))$ corresponding to $S$, which we may assume contains the alcove a we fixed above. We let $W_{\text {aff }}$ denote the affine Weyl group, which is a Coxeter group generated by the reflections through the walls of a. The group $\widetilde{W}$ acts on the set of all alcoves in the apartment of $\mathcal{B}(G(L))$ corresponding to $S$; let $\Omega_{G}=\Omega_{G, \mathbf{a}}$ denote the stabilizer of $\mathbf{a}$. There exists a $\sigma$-equivariant decomposition

$$
\widetilde{W}=W_{\text {aff }} \rtimes \Omega_{G} .
$$


We extend the Bruhat order $\leq$ and the length function $\ell$ from $W_{\text {aff }}$ to $\widetilde{W}$ in the usual way: if $x, x^{\prime} \in \widetilde{W}$ are written as $w \cdot \tau$ and $w^{\prime} \cdot \tau^{\prime}$ via the preceding decomposition, then we set $\ell(x):=\ell(w)$ and declare that $x \leq x^{\prime}$ if and only if both $w \leq w^{\prime}$ and $\tau=\tau^{\prime}$. We can identify $W_{\text {aff }}$ with the Iwahori-Weyl group associated to the pair $G_{\mathrm{sc}}, S_{\mathrm{sc}}$, where $S_{\mathrm{sc}}$ is the pull-back of $\left(S \cap G_{\mathrm{der}}\right)^{\circ}$ via $G_{\mathrm{sc}} \rightarrow G_{\mathrm{der}}$.

We can embed $\widetilde{W}$ set-theoretically into $G(L)$ by choosing a set-theoretic section of the surjective homomorphism $N_{G}(S)(L) \rightarrow \widetilde{W}$. Since $T(L)_{1} \subset \operatorname{ker}\left(\kappa_{G}\right)$, we easily see that the restriction of $\kappa_{G}$ to $\widetilde{W} \hookrightarrow G(L)$ gives a homomorphism

$$
\kappa_{G}: \widetilde{W} \rightarrow X^{*}(Z(\widehat{G}))_{I}
$$

which is surjective and $\sigma$-equivariant and whose kernel is $W_{\text {aff }}$.

\section{Cartan decomposition: Reduction to the key lemma}

Changing slightly the notation of $[\mathrm{HR}$, we set

$$
\widetilde{W}_{K}:=\left(N_{G}(S)(L) \cap K(L)\right) / T(L)_{1} .
$$

We write $\widetilde{W}_{K}^{\sigma}:=\left(\widetilde{W}_{K}\right)^{\sigma}$.

Our starting point is the following fact (see [HR], esp. Remark 9): the map $K(L) n K(L) \mapsto n \in \widetilde{W}$ induces a bijection

$$
K(L) \backslash G(L) / K(L) \cong \widetilde{W}_{K} \backslash \widetilde{W} / \widetilde{W}_{K},
$$

and taking fixed-points under $\sigma$ yields a bijection

$$
K(F) \backslash G(F) / K(F) \cong \widetilde{W}_{K}^{\sigma} \backslash \widetilde{W}^{\sigma} / \widetilde{W}_{K}^{\sigma} .
$$

The Cartan decomposition follows immediately from the key lemma below, which allows us to describe the right-hand side of (3.0.1) in the desired way. To state this we note that the $\sigma$-stable alcove $\mathbf{a}$ is contained in a unique $\sigma$-stable alcove $\mathbf{a}^{M}$ in the apartment $\mathcal{A}_{L}^{M} \subset \mathcal{B}(M(L))$ corresponding to $S$. As before, we define $\Omega_{M} \subset \widetilde{W}_{M}$ to be the stabilizer of $\mathbf{a}^{M}$ under the action of $\widetilde{W}_{M}$ on the alcoves in $\mathcal{A}_{L}^{M}$.

Lemma 3.0.1. (I) There is a tautological isomorphism $\widetilde{W}_{K}^{\sigma} \widetilde{\rightarrow} W(G, A)$ which allows us to view $W(G, A)$ as a subgroup of $\widetilde{W}^{\sigma}$.

(II) There is a decomposition $\widetilde{W}^{\sigma}=\widetilde{W}_{M}^{\sigma} \cdot W(G, A)$, and $W(G, A)$ normalizes $\widetilde{W}_{M}^{\sigma}$.

(III) $W$ e have $W_{M \text {,aff }}^{\sigma}=1$, and hence because of the $\sigma$-equivariant decomposition

$$
\widetilde{W}_{M}=W_{M, \text { aff }} \rtimes \Omega_{M}
$$

we have $\widetilde{W}^{\sigma}=\Omega_{M}^{\sigma} \rtimes W(G, A)$.

The Kottwitz homomorphism gives an isomorphism

$$
\kappa_{M}: \Omega_{M}^{\sigma} \stackrel{\Im}{\rightarrow} X^{*}(Z(\widehat{M}))_{I}^{\sigma}
$$

(cf. [Ko97, 7.7). Putting this together with the lemma we get Theorem 1.0.3.

The proof of Lemma 3.0.1 will occupy the next four sections. 


\section{Some IngREdiEnts ABOUt PARAHORIC SUbGROUPS}

4.1. Parahoric subgroups of $F$-Levi subgroups. As before, let $A$ denote a maximal $F$-split torus in $G$, let $S \supseteq A$ be a maximal $L$-split torus which is defined over $F$, and let $T=C_{G}(S)$ be a maximal torus of $G$ which is defined over $F$.

Let $A_{M}$ denote any subtorus of $A$, and let $M=C_{G}\left(A_{M}\right)$. Thus $M$ is a semistandard $F$-Levi subgroup of $G$. The extended buildings $\mathcal{B}(M(L))$ and $\mathcal{B}(G(L))$ share an apartment (which corresponds to $S$ ), but the affine hyperplanes in the apartment $\mathcal{A}_{L}^{M}$ for $M(L)$ form a subset of those in the apartment $\mathcal{A}_{L}$ for $G(L)$. Hence any facet $\mathbf{a}_{J}$ in $\mathcal{A}_{L}$ is contained in a unique facet in $\mathcal{A}_{L}^{M}$, which we will denote by $\mathbf{a}_{J}^{M}$.

The following result was proved in $[\mathrm{H} 09$ in the special case where $G$ splits over $L$.

Lemma 4.1.1. Suppose $J(L) \subset G(L)$ is the parahoric subgroup corresponding to a facet $\mathbf{a}_{J} \subset \mathcal{A}_{L}$. Then $J(L) \cap M$ is a parahoric subgroup of $M(L)$, and corresponds to the facet $\mathbf{a}_{J}^{M} \subset \mathcal{A}_{L}^{M}$.

Proof. The main result of $[\mathrm{HR}$ is the following characterization of parahoric subgroups:

$$
J(L)=\operatorname{Fix}\left(\mathbf{a}_{J}\right) \cap G(L)_{1}
$$

where $\operatorname{Fix}\left(\mathbf{a}_{J}\right)$ is the set of elements of $G(L)$ that fixes every point of $\mathbf{a}_{J}$. Applying this for the groups $M$ and $G$, we only need to show that

$$
\operatorname{Fix}\left(\mathbf{a}_{J}\right) \cap G(L)_{1} \cap M(L)=\operatorname{Fix}\left(\mathbf{a}_{J}^{M}\right) \cap M(L)_{1} .
$$

The functoriality of the Kottwitz homomorphisms shows $M(L)_{1} \subset G(L)_{1}$, and then the inclusion " $\supseteq$ " is evident. Let $\mathbf{a}^{M}$ denote an alcove in $\mathcal{A}_{L}^{M}$ whose closure contains $\mathbf{a}_{J}^{M}$. Let $I_{M}$ denote the Iwahori subgroup of $M(L)$ corresponding to $\mathbf{a}^{M}$.

Let $S_{\mathrm{sc}}^{M}$, resp., $T_{\mathrm{sc}}^{M}$ denote the pull-back of the torus $\left(S \cap M_{\mathrm{der}}\right)^{\circ}$, resp., $T \cap M_{\mathrm{der}}$ along the homomorphism $M_{\mathrm{sc}} \rightarrow M_{\mathrm{der}}$. To prove the inclusion " $\subseteq$ " it is enough to prove the following claim, since $N_{M_{\mathrm{sc}}}\left(S_{\mathrm{sc}}^{M}\right)(L)$ and $I_{M}$ belong to $M(L)_{1}$. Here and in what follows, we abuse notation slightly by writing $N_{M_{\mathrm{sc}}}\left(S_{\mathrm{sc}}^{M}\right)(L)$ where we really mean its image in $M(L)$.

Claim. Any element $m \in M(L) \cap G(L)_{1}$ which fixes a point in $\mathbf{a}_{J}^{M}$ belongs to

$$
I_{M} N_{M_{\mathrm{sc}}}\left(S_{\mathrm{sc}}^{M}\right)(L) I_{M}
$$

and fixes every point of $\mathbf{a}_{J}^{M}$.

Proof. Recall the decomposition

$$
I_{M} \backslash M(L) / I_{M} \cong N_{M}(S)(L) / T(L)_{1}
$$

of [HR], Prop. 8. Using this we may assume $m \in N_{M}(S)(L)$.

We will show that for such an element $m$ which fixes a point of $\mathbf{a}_{J}^{M}$ we have $m \in T(L)_{1} N_{M_{\mathrm{sc}}}\left(S_{\mathrm{sc}}^{M}\right)(L)$, which will prove the first statement of the claim. It will also prove the second statement, since then $m$ determines a type-preserving automorphism of the apartment $\mathcal{A}_{L}^{M}$, hence fixes every point of $\mathbf{a}_{J}^{M}$ if it fixes any of its points.

Choose a special vertex $\mathbf{a}_{0}^{M}$ contained in the closure of $\mathbf{a}^{M}$, and let $K_{0}$ denote the corresponding special maximal parahoric subgroup of $M(L)$. We may write $m=t n$, where $t \in T(L)$ and $n \in N_{M}(S)(L) \cap K_{0}$ (cf. [HR], Prop. 13). Define $\nu \in X_{*}(T)_{I}$ to be $\kappa_{T}(t)$ and $w \in W(M, S)$ to be the image of $n$ under the projection 
$N_{M}(S)(L) \rightarrow W(M, S)$. Thus $m$ maps to the element $t_{\nu} w \in X_{*}(T)_{I} \rtimes W(M, S) \cong$ $\widetilde{W}_{M}$, the Iwahori-Weyl group for $M$.

Let $\Sigma^{\vee}$ denote the coroots associated to the unique reduced root system $\Sigma$ such that the set of affine roots $\Phi_{\text {af }}(G(L), S)$ on $\mathcal{A}_{L}$ are given by $\Phi_{\text {af }}=\{\alpha+k \mid \alpha \in \Sigma, k \in$ $\mathbb{Z}\}$; cf. $\left[\mathrm{HR}\right.$. Let $\Sigma_{M}^{\vee}$ denote the coroots for the corresponding root system $\Sigma_{M}$ for $\Phi_{\mathrm{af}}(M(L), S)$ on $\mathcal{A}_{L}^{M}$. Let $Q^{\vee}(\Sigma)$, resp., $Q^{\vee}\left(\Sigma_{M}\right)$ denote the lattice spanned by $\Sigma^{\vee}$, resp., $\Sigma_{M}^{\vee}$. Recall from $\left[\mathrm{HR}\right.$ ] that we have identifications $Q^{\vee}(\Sigma) \cong X_{*}\left(T_{\mathrm{sc}}\right)_{I}$ and $Q^{\vee}\left(\Sigma_{M}\right) \cong X_{*}\left(T_{\mathrm{sc}}^{M}\right)_{I}$. Also, we have $\Phi_{\mathrm{af}}(M(L), S) \subseteq \Phi_{\mathrm{af}}(G(L), S)$, and therefore $Q^{\vee}\left(\Sigma_{M}\right) \subseteq Q^{\vee}(\Sigma)$

Clearly, $w$ is the image of an element from $N_{M_{\mathrm{sc}}}\left(S_{\mathrm{sc}}^{M}\right)(L) \cap K_{0}$, since the latter also surjects onto $W(M, S)$. Thus we need only show that $\nu \in Q^{\vee}\left(\Sigma_{M}\right)$, since $Q^{\vee}\left(\Sigma_{M}\right)$ is also in the image of $N_{M_{\mathrm{sc}}}\left(S_{\mathrm{sc}}^{M}\right)(L) \rightarrow \widetilde{W}_{M}$.

First, we will prove that $\nu \in Q^{\vee}(\Sigma)$. Indeed, by construction $t \in G(L)_{1}$, and using

$$
X_{*}(T)_{I} / X_{*}\left(T_{\mathrm{sc}}\right)_{I} \cong X^{*}(Z(\widehat{G}))_{I}
$$

(cf. [HR]) we see that $\nu \in X_{*}\left(T_{\mathrm{sc}}\right)_{I}=Q^{\vee}(\Sigma)$.

Next, let $r$ denote the order of $w \in W(M, S)$. The element $m^{r}$ maps to $\left(t_{\nu} w\right)^{r} \in$ $\widetilde{W}_{M}$, which is the translation by the element $\mu:=\sum_{i=0}^{r-1} w^{i} \nu \in Q^{\vee}(\Sigma)$. But as this translation fixes a point of $\mathbf{a}_{J}^{M}$, we must have $\mu=0$. Since $w^{i} \nu \equiv \nu$ modulo $Q^{\vee}\left(\Sigma_{M}\right)$, it follows that

$$
\nu \in Q^{\vee}\left(\Sigma_{M}\right)_{\mathbb{Q}} \cap Q^{\vee}(\Sigma)=Q^{\vee}\left(\Sigma_{M}\right) .
$$

This completes the proof of the claim, and thus the lemma.

4.2. Parahoric subgroups of minimal F-Levi subgroups. Now we return to the usual notation, where $M:=C_{G}(A)$ is a minimal $F$-Levi subgroup of $G$. In this case $M_{\mathrm{ad}}$ is anisotropic over $F$, so the semi-simple building $\mathcal{B}\left(M_{\mathrm{ad}}(F)\right)=$ $\mathcal{B}\left(M_{\text {ad }}(L)\right)^{\sigma}$ is a singleton and the apartment $\left(\mathcal{A}_{L}^{M}\right)^{\sigma}$ contains no affine hyperplanes. Therefore, $M(F)$ has only one parahoric subgroup.

Lemma 4.2.1. Let $J$ be any parahoric subgroup of $G(L)$ corresponding to a $\sigma$ invariant facet $\mathbf{a}_{J}$ in $\mathcal{A}_{L}$. Then $J(L) \cap M(F)=M(F)_{1}$.

Proof. By Lemma 4.1.1, the inclusion " $\subseteq$ " is clear. Let $m \in M(F)_{1}$. Since $m$ acts trivially on the apartment $\mathcal{A}_{L}^{\sigma}$ in the building $\mathcal{B}(G(F))=\mathcal{B}(G(L))^{\sigma}$, it fixes a point of the $\sigma$-invariant facet $\mathbf{a}_{J}$ (e.g. its barycenter). But then since $m \in G(F)_{1}$, by the Claim in the proof of Lemma 4.1.1 (taking $M=G$ ), $m$ fixes every point in $\mathbf{a}_{J}$. Clearly, then $m \in \operatorname{Fix}\left(\mathbf{a}_{J}\right) \cap G(L)_{1} \cap M(F)=J(L) \cap M(F)$.

Lemma 4.2.2. Let $K(L)$ denote the parahoric subgroup of $G(L)$ whose $\sigma$-fixed subgroup $K=K(L)^{\sigma}$ is the special maximal compact subgroup of $G(F)$ we fixed earlier. Then

$$
K \cap N_{G}(S)(L) \cap M(F)=T(F)_{1} .
$$

Proof. Fix an Iwahori subgroup $I \subset G(L)$ corresponding to a $\sigma$-invariant alcove in $\mathcal{A}_{L}$. Note that by Lemma 4.2.1 we have $K \cap M(F)=I \cap M(F)$ and hence

$$
K \cap N_{G}(S)(L) \cap M(F)=I \cap N_{G}(S)(L) \cap M(F) .
$$

By $\left[\mathrm{HR}\right.$, Lemma 6, the right-hand side is $T(L)_{1} \cap M(F)=T(F)_{1}$. 


\section{The isomorphism $\widetilde{W}_{K}^{\sigma} \cong W(G, A)$}

By $\left[\mathrm{HR}\right.$, Remark 9 , any element of $\widetilde{W}_{K}^{\sigma}$ is represented by an element of $N_{G}(S)(F)$. Let $x \in N_{G}(S)(F)$. Then $x S x^{-1}=S$ contains $x A x^{-1}$ and $A$, which being maximal $F$-split tori in $S$, must coincide. Thus, there is a tautological homomorphism

$$
N_{G}(S)(F) \rightarrow N_{G}(A)(F) .
$$

By Lemma 4.2.2, this factors to give an injective homomorphism

$$
\widetilde{W}_{K}^{\sigma} \hookrightarrow W(G, A) .
$$

The next statement furnishes the proof of Lemma 3.0.1, (I).

Lemma 5.0.1. The homomorphism $\widetilde{W}_{K}^{\sigma} \rightarrow W(G, A)$ is an isomorphism. This allows us to regard $W(G, A)$ as a subgroup of $\widetilde{W}^{\sigma}$.

Proof. It is enough to prove the domain and codomain have the same order. Let $k_{L}$ denote the residue field of $\mathcal{O}_{L}$, which can be identified with an algebraic closure of $k_{F}$. Consider the special fiber $\overline{\mathcal{G}}_{\mathbf{a}_{0}}^{\circ}=\mathcal{G}_{\mathbf{a}_{0}}^{\circ} \times_{\mathcal{O}_{L}} k_{L}$ of the Bruhat-Tits group scheme $\mathcal{G}_{\mathbf{a}_{0}}^{\circ}$ over $\mathcal{O}_{L}$ which is associated to the facet $\mathbf{a}_{0}$ in the building $\mathcal{B}(G(L))$. Let $\overline{\mathcal{G}}_{\mathbf{a}_{0}}^{\circ \text { red }}$ denote the maximal reductive quotient of $\overline{\mathcal{G}}_{\mathbf{a}_{0}}^{\circ}$. By [HR], Prop. 12, $\widetilde{W}_{K}$ is the Weyl group of $\overline{\mathcal{G}}_{\mathbf{a}_{0}}^{\circ \text {,red }}$. The group $\overline{\mathcal{G}}_{\mathbf{a}_{0}}^{\circ \text {,red }}$ is defined over $k_{F}$, and in fact we have $\overline{\mathcal{G}}_{\mathbf{a}_{0}}^{\circ \text {,red }}=\overline{\mathcal{G}}_{v_{F}}^{\circ \text { red }} \times_{k_{F}} k_{L}$, where $\overline{\mathcal{G}}_{v_{F}}^{\circ}$ is the special fiber of $\mathcal{G}_{v_{F}}^{\circ}$ (cf. Land, Cor. 10.10). Since $k_{F}$ is finite, $\overline{\mathcal{G}}_{v_{F}}^{\circ \text {,red }}$ is automatically quasi-split over $k_{F}$, and it follows that $\widetilde{W}_{K}^{\sigma}$ is the Weyl group of $\overline{\mathcal{G}}_{v_{F}}^{\circ \text {,red }}$ (this is well known, but one can also use the argument which yields Remark 6.1.3 below).

On the other hand, by Tits, 3.5.1, the root system of $\overline{\mathcal{G}}_{v_{F}}^{\circ \text {,red }}$ is $\Phi_{v_{F}}$, the root system consisting of the vector parts of the affine roots for $A$ which vanish on $v_{F}$ (loc. cit. 1.9). Because $v_{F}$ is special, $\Phi_{v_{F}}=\Phi(G, A)$, the relative root system. Thus the Weyl group of $\overline{\mathcal{G}}_{v_{F}}^{\circ \text {,red }}$ is isomorphic to $W(G, A)$.

These remarks imply that $\widetilde{W}_{K}^{\sigma}$ and $W(G, A)$ are abstractly isomorphic groups and, in particular, they have the same order.

\section{A DeCOMposition of the IWAHORI Weyl group}

The goal here is to prove Lemma 3.0.1 (II).

6.1. A lemma on finite Weyl groups. Let $w \in W(G, A)$ and choose a representative $g \in N_{G}(A)(F)$ for $w$; write $[g]=w$. The tori $g S g^{-1}$ and $S$ are both maximal $L$-split tori in $M$, hence there exists $m \in M(L)$ such that $m g S g^{-1} m^{-1}=S$. We claim that the map

$$
\begin{aligned}
W(G, A) & \rightarrow W(G, S) / W(M, S) \\
w & \mapsto[m g] \cdot W(M, S)
\end{aligned}
$$

is well defined and injective. Indeed, suppose $g_{0} \in N_{G}(A)(F)$ represents an element $w_{0} \in W(G, A)$ and that $m_{0} \in M(L)$ satisfies $m_{0} g_{0} S g_{0}^{-1} m_{0}^{-1}=S$. To show the map is well defined, we suppose $w=w_{0}$ and we show that $(m g)^{-1} m_{0} g_{0} \in$ $N_{M}(S)$. It will suffice to show $(m g)^{-1} m_{0} g_{0}$ belongs to $M(L)$. Since $g$ normalizes $M=C_{G}(A)$ and $g^{-1} g_{0} \in M$, this is obvious. To show the map is injective we suppose $[m g] W(M, S)=\left[m_{0} g_{0}\right] W(M, S)$, that is, $(m g)^{-1} m_{0} g_{0} \in N_{M}(S)$. Arguing 
as before, we deduce that $g^{-1} g_{0} \in M$. This shows that $w=w_{0}$ and so we get the injectivity.

Remark 6.1.1. Here is another way to describe the map. For an element $w \in$ $W(G, A)$, using Lemma 5.0.1 choose an element $x \in N_{G}(S)(F) \cap K$ whose image in $\widetilde{W}_{K}^{\sigma}$ maps to $w$ under the isomorphism $\widetilde{W}_{K}^{\sigma} \widetilde{\rightarrow} W(G, A)$. Then the map sends $w$ to the coset $[x] W(M, S)$.

Lemma 6.1.2. The above map induces a bijection

$$
W(G, A) \stackrel{\Im}{\rightarrow}[W(G, S) / W(M, S)]^{\sigma} .
$$

Proof. First we prove the image $[m g] W(M, S)$ is $\sigma$-invariant. This follows because the element $(m g)^{-1} \sigma(m) g$ belongs to $M$, hence to $N_{M}(S)$.

Next, we prove the surjectivity. Suppose $x \in N_{G}(S)$ projects to an element in $W(G, S)$ which represents a $\sigma$-fixed coset $C$ in $W(G, S) / W(M, S)$, that is, $x^{-1} \sigma(x) \in M$. Then the subtorus $x A x^{-1} \subset S$ is defined over $F$. The inner automorphism $\operatorname{Int}(x): S \rightarrow S$, restricted to $A$ gives an isomorphism $\operatorname{Int}(x): A \widetilde{\Im} A x^{-1}$ which is defined over $F$. It follows that $x A x^{-1}$ is $F$-split. Since $A$ and $x A x^{-1}$ are maximal $F$-split tori in $S$, they coincide. Thus $x \in N_{G}(A)$, and the image of $x$ is the coset $C$.

Remark 6.1.3. If $G$ is quasi-split over $F$, then $M=T$ and we recover the well-known result that $W(G, A)=W(G, S)^{\sigma}$.

6.2. Proof of the decomposition. We keep the notation of the previous subsection. There is a commutative diagram of exact sequences with $\sigma$-equivariant morphisms and injective vertical maps

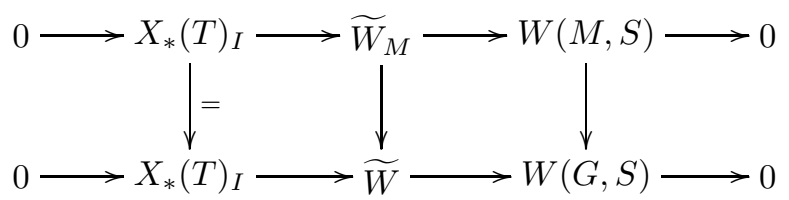

(see $\left[\mathrm{HR}\right.$, Prop. 13). The canonical map $\widetilde{W}_{M} \backslash \widetilde{W} \rightarrow W(M, S) \backslash W(G, S)$ is bijective and $\sigma$-equivariant, so we get

$$
\left[\widetilde{W}_{M} \backslash \widetilde{W}\right]^{\sigma} \cong[W(M, S) \backslash W(G, S)]^{\sigma}
$$

Using the map $W(G, A) \hookrightarrow \widetilde{W}^{\sigma}$ constructed in Lemma 5.0.1 we get a commutative diagram

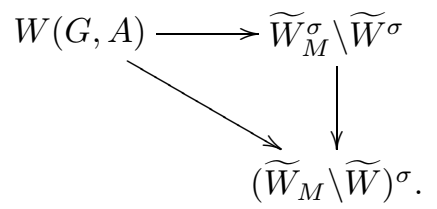

The commutativity of this diagram follows using Remark 6.1.1. Since the diagonal arrow is a bijection by the above discussion, and the vertical arrow is obviously an injection, it follows that all arrows in the diagram are bijections. The decomposition

$$
\widetilde{W}^{\sigma}=\widetilde{W}_{M}^{\sigma} \cdot W(G, A)
$$

follows. It is clear that $W(G, A)$ normalizes $\widetilde{W}_{M}^{\sigma}$. This completes the proof of Lemma 3.0.1, (II) . 


\section{END OF PROOF of THE CARTAN DECOMPOSITION}

\subsection{Invariants in the affine Weyl group of $M$.}

Lemma 7.1.1. Let $M$ again denote a minimal $F$-Levi subgroup, and let $W_{M \text {,aff }}$ denote the affine Weyl group associated to $M$. Then $W_{M, \text { aff }}^{\sigma}=1$.

Proof. We identify $W_{M, \text { aff }}$ with the Iwahori-Weyl group $N_{M_{\mathrm{sc}}}\left(S_{\mathrm{sc}}^{M}\right)(L) / T_{\mathrm{sc}}^{M}(L)_{1}$. Let $I_{M_{\mathrm{sc}}}$ denote the Iwahori subgroup of $M_{\mathrm{sc}}(L)$ corresponding to a $\sigma$-invariant alcove $\mathbf{a}^{M_{\mathrm{sc}}}$ in the apartment $\mathcal{A}_{L}^{M_{\mathrm{sc}}}=X_{*}\left(S_{\mathrm{sc}}^{M}\right)_{\mathbb{R}}$ of $\mathcal{B}\left(M_{\mathrm{sc}}(L)\right)$ associated to the torus $S_{\mathrm{sc}}^{M}$. By [HR], Remark 9 , the set $W_{M \text {,aff }}^{\sigma}$ is in bijective correspondence with

$$
I_{M_{\mathrm{sc}}}(F) \backslash M_{\mathrm{sc}}(F) / I_{M_{\mathrm{sc}}}(F) .
$$

Therefore, it is enough to prove that $M_{\mathrm{sc}}(F)=I_{M_{\mathrm{sc}}}(F)$. But $M_{\mathrm{sc}}(F)=M_{\mathrm{sc}}(F)_{1}=$ $I_{M_{\mathrm{sc}}}$. (For the second equality, use Lemma 4.2.1 with $G=M_{\mathrm{sc}}$.)

7.2. Conclusion of the proof of Theorem 1.0.3. We have fixed the $\sigma$-stable alcove $\mathbf{a}$ and this determines the $\sigma$-stable alcove $\mathbf{a}^{M}$ and the corresponding subgroup $\Omega_{M} \subset \widetilde{W}_{M}$. There is a canonical $\sigma$-equivariant decomposition $\widetilde{W}_{M}=W_{M \text {,aff }} \rtimes \Omega_{M}$, so in view of the above lemma, we deduce that

$$
\widetilde{W}_{M}^{\sigma}=\Omega_{M}^{\sigma} .
$$

This completes the proof of the last part, namely (III), of Lemma 3.0.1. Since the Theorem 1.0 .3 is a consequence of Lemma 3.0.1, we have proved Theorem 1.0 .3 .

\section{Characterization of special maximal compact subgroups}

Let

$$
v_{G}: G(L) \rightarrow X^{*}(Z(\widehat{G}))_{I} / \text { torsion }
$$

denote the homomorphism derived from the Kottwitz homomorphism

$$
\kappa_{G}: G(L) \rightarrow X^{*}(Z(\widehat{G}))_{I}
$$

in the obvious way. Denote its kernel by $G(L)^{1}$ and let $G(F)^{1}=G(L)^{1} \cap G(F)$ (cf. BT2, 5.1.29). Note that if $M$ is a minimal $F$-Levi subgroup of $G$, then $M(F)^{1}$ is the unique maximal compact open subgroup of $M(F)$, consistent with the notation used in the introduction.

Let $K:=\mathcal{G}_{v_{F}}^{\circ}\left(\mathcal{O}_{F}\right)$, the maximal parahoric subgroup of $G(F)$ corresponding to $v_{F}$. By $[\mathrm{HR}$, Prop. 3 and Remark 9, we have the equality

$$
K=G(F)_{1} \cap \operatorname{Fix}\left(\mathbf{a}_{0}\right) .
$$

Using the Claim from the proof of Lemma 4.1.1 in the case $M=G$, we derive the equality

$$
K=G(F)_{1} \cap \operatorname{Fix}\left(v_{F}\right) .
$$

Our goal is to prove the analogous description of $\widetilde{K}$.

Lemma 8.0.1. The special maximal compact subgroups of $G(F)$ are precisely the subgroups of the form

$$
\widetilde{K}=G(F)^{1} \cap \operatorname{Fix}\left(v_{F}\right),
$$

where $v_{F}$ ranges over the special vertices in the building $\mathcal{B}\left(G_{\mathrm{ad}}(F)\right)$. 
Proof. A compact subgroup of $G(F)$ is automatically contained in $G(F)^{1}$. This follows from the alternative description of $G(L)^{1}$ as the intersection of the kernels of the homomorphisms $|\chi|: G(L) \rightarrow \mathbb{R}_{>0}$, where $\chi$ ranges over $L$-rational characters on $G$.

Thus, using BT1, Cor. (4.4.1), every maximal compact subgroup $\widetilde{K}$ of $G(F)$ (equiv., of $G(F)^{1}$ ) is the stabilizer in $G(F)^{1}$ of a well-defined facet in the building $\mathcal{B}\left(G_{\text {der }}(F)\right)$. By definition, such a $\widetilde{K}$ is special if and only if the facet it stabilizes is a special vertex $v_{F}$. In that case, we have $\widetilde{K}=G(F)^{1} \cap \operatorname{Fix}\left(v_{F}\right)$.

To show the converse, we must check that $G(F)^{1} \cap \operatorname{Fix}\left(v_{F}\right)$ is compact (the argument above will then show it is (special) maximal compact). Recall $K=$ $\mathcal{G}_{v_{F}}^{\circ}\left(\mathcal{O}_{F}\right)$ is compact and is given by (8.0.1). Since $G(F)_{1} \cap \operatorname{Fix}\left(v_{F}\right)$ has finite index in $G(F)^{1} \cap \operatorname{Fix}\left(v_{F}\right)$, and since the former is compact, so is the latter. This completes the proof.

Remark 8.0.2. Equation (8.0.1) can be generalized. Let $\mathbf{a}_{J}$ denote any $\sigma$-stable alcove in $\mathcal{B}(G(L))$. Then

$$
\mathcal{G}_{\mathbf{a}_{J}}^{\circ}\left(\mathcal{O}_{F}\right)=G(F)_{1} \cap \operatorname{Fix}\left(\mathbf{a}_{J}^{\sigma}\right) .
$$

However, if $\mathcal{G}_{\mathbf{a}_{J}}^{\circ}$ is replaced with the "full-fixer" group scheme $\widehat{\mathcal{G}}_{\mathbf{a}_{J}}$ (cf. BT2, 4.6.28, 5.1.29), the corresponding statement

$$
\widehat{\mathcal{G}}_{\mathbf{a}_{J}}\left(\mathcal{O}_{F}\right)=G(F)^{1} \cap \operatorname{Fix}\left(\mathbf{a}_{J}^{\sigma}\right)
$$

is false. Indeed, the right-hand side, a general analogue of our $\widetilde{K}$ above, can be strictly larger than the left-hand side. For example, consider the anisotropic group $G=D^{\times} / F^{\times}$of Remark 11.1.3, and let $\mathbf{a}_{J}^{\sigma}=v_{F}$, and $\mathbf{a}_{J}=\mathbf{a}$. Then the right-hand side is $G(F)$, but the left-hand side is a subgroup of index $n=\sqrt{\operatorname{dim}_{F}(D)}$, namely $\mathcal{O}_{D}^{\times} / \mathcal{O}_{F}^{\times}$

\section{Statement of the Satake isomorphism}

In this section, let $P=M N$ denote any $F$-rational parabolic subgroup of $G$ with unipotent radical $N$, which has $M$ as a Levi factor.

9.1. Iwasawa decomposition. In light of Lemma 8.0.1, the following version of the Iwasawa decomposition can be derived easily from similar statements in the literature (cf. [BT1, Rem. (4.4.5) or Prop. (7.3.1)):

Proposition 9.1.1. There is an equality of sets

$$
G(F)=P(F) \cdot \widetilde{K}(F) .
$$

We need the variant of this where $\widetilde{K}(F)$ is replaced by $K(F)$. It will be enough to prove that

$$
\widetilde{K}(F)=(\widetilde{K} \cap M(F)) \cdot K(F) .
$$

Using (3.0.1) together with Lemma 3.0.1, we see that any element $\tilde{k} \in \widetilde{K}(F)$ satisfies

$$
\tilde{k} \in K(F) m K(F)
$$

for some $m \in \Omega_{M}^{\sigma} \subset M(F)$. It follows that $m \in \widetilde{K}(F)$, and then since $\widetilde{K}(F)$ normalizes $K(F)$ (cf. e.g. Lemma 8.0.1), we see that $\tilde{k} \in m K(F)$ as desired.

We have thus proved the first part of the following corollary. 
Corollary 9.1.2 (Iwasawa decomposition). There is an equality of sets

$$
G(F)=P(F) \cdot K(F) .
$$

Moreover, $P(F) \cap K(F)=(M(F) \cap K) \cdot(N(F) \cap K)$.

Proof. We need only show the second equality, which can be rewritten as

$$
P(F) \cap \mathcal{G}_{v_{F}}^{\circ}\left(\mathcal{O}_{F}\right)=\left(M(F) \cap \mathcal{G}_{v_{F}}^{\circ}\left(\mathcal{O}_{F}\right)\right) \cdot\left(N(F) \cap \mathcal{G}_{v_{F}}^{\circ}\left(\mathcal{O}_{F}\right)\right) .
$$

This follows from [BT2, 5.2.4 (taking the set denoted by $\Omega$ there to be $\left\{v_{F}\right\}$ ).

9.2. Construction of the Satake transform. We will follow the approach taken in [HKP], which treated the case of $F$-split groups.

Recall that $\mathcal{H}_{K}:=C_{c}(K(F) \backslash G(F) / K(F))$, the spherical Hecke algebra of $K(F)$ bi-invariant compactly-supported functions on $G(F)$. The convolution is defined using the Haar measure on $G(F)$ which gives $K(F)$ volume 1.

Set $R:=\mathbb{C}\left[M(F) / M(F)_{1}\right]$. Since $M(F)_{1}$ is the unique parahoric subgroup of $M(F)$, this is just the Iwahori-Hecke algebra for $M(F)$. Let

$$
\mathbf{M}:=C_{c}\left(M(F)_{1} N(F) \backslash G(F) / K(F)\right),
$$

where the subscript "c" means we consider functions supported on finitely many double cosets. Then $\mathbf{M}$ carries an obvious right convolution action under $\mathcal{H}_{K}$. It also carries a left action by $R$ given by normalized convolutions:

$$
r \cdot \phi(m):=\int_{M(F)} \delta_{P}^{1 / 2}\left(m_{1}\right) r\left(m_{1}\right) \phi\left(m_{1}^{-1} m\right) d m_{1} .
$$

Here $d m_{1}$ is the Haar measure on $M(F)$ giving $M(F)_{1}$ volume 1 , and $\delta_{P}$ is the modular function on $P(F)$ given by the normalized absolute value of the determinant of the adjoint action on $\operatorname{Lie}(N(F))$. For $m \in M(F)$ we have

$$
\delta_{P}(m):=|\operatorname{det}(\operatorname{Ad}(m) ; \operatorname{Lie}(N(F)))|_{F} .
$$

The actions of $R$ and $\mathcal{H}_{K}$ on $\mathbf{M}$ commute, so that $\mathbf{M}$ is an $\left(R, \mathcal{H}_{K}\right)$-bimodule.

Lemma 9.2.1. The R-module $\mathbf{M}$ is free of rank 1, with canonical generator

$$
v_{1}:=\operatorname{char}\left(M(F)_{1} N(F) K(F)\right) .
$$

Proof. This follows directly from Corollary 9.1.2,

Given $f \in \mathcal{H}_{K}$, let $f^{\vee} \in R$ denote the unique element satisfying the identity

$$
v_{1} f=f^{\vee} v_{1} .
$$

It is obvious that

$$
\begin{aligned}
\mathcal{H}_{K} & \rightarrow R \\
f & \mapsto f^{\vee}
\end{aligned}
$$

is a $\mathbb{C}$-algebra homomorphism.

Evaluating both sides of (9.2.1) on $m \in M(F)$ and using the usual $G=M N K$ integration formula (see [Car]), we get the familiar expression

$$
f^{\vee}(m)=\delta_{P}^{-1 / 2}(m) \int_{N(F)} f(n m) d n=\delta_{P}^{1 / 2}(m) \int_{N(F)} f(m n) d n,
$$

where $d n$ gives $N(F) \cap K(F)$ measure 1 . 


\section{The SATAKE TRANSFORM IS AN ISOMORPHISM}

10.1. Weyl group invariance. The first step is to prove that $f^{\vee}$ belongs to the subring $R^{W(G, A)}$ of $W(G, A)$-invariants in $R$. Once this is proved, the functoriality of the Kottwitz homomorphism

$$
\kappa_{M}: M(F) / M(F)_{1} \widetilde{\rightarrow} X^{*}(Z(\widehat{M}))_{I}^{\sigma}
$$

shows that $f^{\vee} \in \mathbb{C}\left[X^{*}(Z(\widehat{M}))_{I}^{\sigma}\right]^{W(G, A)}$, as well.

The argument is virtually the same as Cartier's Car. Define a function on $m \in M(F)$ by

$$
D(m)=\mid\left.\operatorname{det}(\operatorname{Ad}(m)-1 ; \text { Lie } G(F) / \text { Lie } M(F))\right|^{1 / 2} .
$$

Then exactly as in loc. cit. one can prove the formula

$$
f^{\vee}(m)=D(m) \int_{G / A} f\left(g m g^{-1}\right) \frac{d g}{d a}
$$

on the Zariski-dense subset of elements $m \in M(F)$ which are regular semi-simple as elements in $G$. Here $d g$ (resp. $d a$ ) is the Haar measure on $G(F)$ (resp. $A(F)$ ) which gives $K$ (resp. $K \cap A(F)$ ) volume 1. By Lemma 3.0.1 (I), every element $w \in W(G, A)$ can be represented by an $x \in N_{G}(A) \cap K$. Clearly, $D(m)=$ $D\left(x m x^{-1}\right)$. Since the measure on $G / A$ is invariant under conjugation by $x$, we see as in loc. cit. that the integral in (10.1.1) is also invariant under $m \mapsto x m x^{-1}$. Thus (10.1.1) is similarly invariant, as desired.

Remark 10.1.1. As in the case of $\mathcal{H}_{\widetilde{K}}$, equation (10.1.1) also shows that $f^{\vee}$ is independent of the choice of $F$-rational parabolic subgroup $P$ which contains $M$ as a Levi factor.

10.2. Upper triangularity. The second step is to show that with respect to natural $\mathbb{C}$-bases of $\mathcal{H}_{K}$ and $R^{W(G, A)}$, the map $f \mapsto f^{\vee}$ is "invertible upper triangular", hence is an isomorphism of algebras.

The set $\widetilde{W}_{K}^{\sigma} \backslash \widetilde{W}^{\sigma} / \widetilde{W}_{K}^{\sigma} \cong W(G, A) \backslash \Omega_{M}^{\sigma}$ provides a natural $\mathbb{C}$-basis for $\mathcal{H}_{K}$ and for $R^{W(G, A)}$. Recall that $\widetilde{W}$ has a natural structure of a quasi-Coxeter group

$$
\widetilde{W}=W_{\text {aff }} \rtimes \Omega
$$

(cf. [HR], Lemma 14). We extend the Bruhat order $\leq$ and the length function $\ell$ from $W_{\text {aff }}$ to $\widetilde{W}$ in the usual way (cf. loc. cit.). Given $x \in \widetilde{W}$, denote by $\tilde{x} \in \widetilde{W}$ the unique minimal element in $\widetilde{W}_{K} x \widetilde{W}_{K}$. (Note that $\widetilde{W}_{K}$ is finite and that the usual theory of such minimal elements for Coxeter groups goes over to handle quasi-Coxeter groups.)

By $\mathrm{HR}$, Remark 9, we may regard $\widetilde{W}_{K}^{\sigma} \backslash \widetilde{W}^{\sigma} / \widetilde{W}_{K}^{\sigma}$ as a subset (the $\sigma$-invariant elements) in $\widetilde{W}_{K} \backslash \widetilde{W} / \widetilde{W}_{K}$. For $y, y^{\prime} \in W(G, A) \backslash \Omega_{M}^{\sigma}$, resp., $x, x^{\prime} \in \widetilde{W}_{K}^{\sigma} \backslash \widetilde{W}^{\sigma} / \widetilde{W}_{K}^{\sigma}$, we define the partial order $\preceq$ by requiring

$$
\begin{aligned}
& y \preceq y^{\prime} \Leftrightarrow \tilde{y} \leq \tilde{y}^{\prime}, \quad \text { resp. } \\
& x \preceq x^{\prime} \Leftrightarrow \tilde{x} \leq \tilde{x}^{\prime} .
\end{aligned}
$$

The set $W(G, A) \backslash \Omega_{M}^{\sigma}$ is countable and every element $y$ has only finitely many predecessors with respect to the partial order $\preceq$. Therefore, there is a total ordering $y_{1}, y_{2}, \ldots$ on this set which is compatible with $\preceq$, meaning that $y_{i} \preceq y_{j}$ only if 
$i \leq j$. Similar remarks apply to the partially ordered set $\widetilde{W}_{K}^{\sigma} \backslash \widetilde{W}^{\sigma} / \widetilde{W}_{K}^{\sigma}$, and we get an analogous total ordering $x_{1}, x_{2}, \ldots$ for it.

We claim that the matrix for $f \mapsto f^{\vee}$ in terms of the bases $\left\{y_{i}\right\}_{1}^{\infty}$ and $\left\{x_{i}\right\}_{1}^{\infty}$ is upper triangular and invertible. The upper triangularity is the content of the next lemma.

Lemma 10.2.1. Suppose $x \in \widetilde{W}^{\sigma}$ and $y \in \Omega_{M}^{\sigma}$ and that

$$
N(F) y K(F) \cap K(F) x K(F) \neq \emptyset .
$$

Then $\tilde{y} \leq \tilde{x}$.

Proof. Let $I$ denote the Iwahori subgroup of $G(L)$ associated to the $\sigma$-stable alcove a, as defined earlier. We shall need two BN-pair relations. The first is the relation

$$
K(L)=I(L) \widetilde{W}_{K} I(L) .
$$

This follows easily using [HR], Prop. 8. The second is the relation

$$
I(L) w I(L) w^{\prime} I(L) \subseteq \coprod_{w^{\prime \prime} \leq w^{\prime}} I(L) w w^{\prime \prime} I(L) .
$$

This relation per se does not appear in the literature, but it follows easily from the BN-pair relations established in [BT2, 5.2 .12 (cf. [HR], paragraph following Lemma 17).

Using (10.2.2) and (10.2.3) we see that (10.2.1) implies that

$$
N(L) y I(L) \cap I(L) x^{\prime} I(L) \neq \emptyset
$$

for some $x^{\prime} \in \widetilde{W}_{K} x \widetilde{W}_{K}$. Write

$$
n y=i x^{\prime} i^{\prime}
$$

for $n \in N(L)$, and $i, i^{\prime} \in I(L)$. Choose a cocharacter $\lambda \in X_{*}(A)$ such that $\varpi^{\lambda} n \varpi^{-\lambda} \in I(L)$. Then multiplying (10.2.5) by $\varpi^{\lambda}$ we see that

$$
I(L) \varpi^{\lambda} y I(L) \subseteq I(L) \varpi^{\lambda} I(L) x^{\prime} I(L) .
$$

Using (10.2.3) again we deduce that

$$
I(L) \varpi^{\lambda} y I(L)=I(L) \varpi^{\lambda} x^{\prime \prime} I(L)
$$

and hence $y=x^{\prime \prime}$ for some $x^{\prime \prime} \in \widetilde{W}$ with $x^{\prime \prime} \leq x^{\prime}$. Thus $\tilde{y} \leq x^{\prime}$. A standard argument then shows that $\tilde{y} \leq \tilde{x}$, which is what we wanted to prove.

Finally, the invertibility follows from the obvious fact that

$$
N(F) x K(F) \cap K(F) x K(F) \neq \emptyset .
$$

This completes the proof that $f \mapsto f^{\vee}$ is an isomorphism.

\section{The Structure of $\Lambda_{M}$}

It is clear that $\Lambda_{M}=X^{*}(Z(\widehat{M}))_{I}^{\sigma}$ is a finitely-generated abelian group. In this section we make it more concrete in various situations. 
11.1. General results. As before, in this subsection $T$ denotes the centralizer in $G$ of the torus $S$. Recall that we can assume $S$ is defined over $F$, and so $T$ is also defined over $F$. Recall also that $T_{\mathrm{sc}}^{M}$ denotes the pull-back of $T$ via $M_{\mathrm{sc}} \rightarrow M$.

Lemma 11.1.1. There is an embedding $X_{*}(T)_{I}^{\sigma} \hookrightarrow \Lambda_{M}$ whose cokernel is isomorphic to the finite abelian group $\operatorname{ker}\left[X_{*}\left(T_{\mathrm{sc}}^{M}\right)_{\Gamma} \rightarrow X_{*}(T)_{\Gamma}\right]$.

Proof. Use the long exact sequence for $H^{i}(\langle\sigma\rangle,-)$ associated to the short exact sequence

$$
0 \longrightarrow X_{*}\left(T_{\mathrm{sc}}^{M}\right)_{I} \longrightarrow X_{*}(T)_{I} \longrightarrow X^{*}(Z(\widehat{M}))_{I} \longrightarrow 0 .
$$

(For a discussion of this short exact sequence, see [HR], proof of Prop. 13.) Note that $X_{*}\left(T_{\mathrm{sc}}^{M}\right)_{I}^{\sigma} \subset W_{M \text {,aff }}^{\sigma}=1$ (cf. Lemma 7.1.1). Also, $X_{*}\left(T_{\mathrm{sc}}^{M}\right)_{\Gamma}$ is finite because $M_{\mathrm{sc}}$ is anisotropic over $F$. The lemma follows easily using these remarks.

Corollary 11.1.2. (a) If $G$ is quasi-split over $F$, then $\Lambda_{M}=X_{*}(T)_{I}^{\sigma}$.

(b) If $G$ is split over $L$, then $\Lambda_{M}$ fits into the exact sequence

$$
1 \rightarrow X_{*}(A) \rightarrow \Lambda_{M} \rightarrow \operatorname{ker}\left[X_{*}\left(T_{\mathrm{sc}}^{M}\right)_{\sigma} \rightarrow X_{*}(T)_{\sigma}\right] \rightarrow 0 .
$$

(c) If $G$ is unramified over $F$, then $\Lambda_{M}=X_{*}(A)$.

Proof. Part (a). Since $G$ is quasi-split over $F$, we have $M=T$, and the desired formula follows directly from the definition of $\Lambda_{M}$.

Part (b) follows immediately from Lemma 11.1.1

Part (c) follows as a special case of either (a) or (b). Part (c) was known previously (cf. [Bo], 9.5).

Remark 11.1.3. If $G$ is semi-simple and anisotropic, then $\Lambda_{M}$ is finite. There are examples, namely $G=D^{\times} / F^{\times}$for $D$ a central simple division algebra over $F$ with $\operatorname{dim}_{F}(D)>1$, where $\Lambda_{M} \neq 0$.

At the opposite extreme, let $E / F$ denote a finite totally ramified extension. Consider the "diagonal" embedding $\mathbb{G}_{m} \hookrightarrow \mathrm{R}_{E / F} \mathbb{G}_{m}$ and set $G=\left(\mathrm{R}_{E / F} \mathbb{G}_{m}\right) / \mathbb{G}_{m}$. Then $\Lambda_{G}$ is torsion, and non-zero if $E \neq F$.

The next proposition tells us how to measure the difference between the subgroups $K$ and $\widetilde{K}$ of $G(F)$ attached to a special vertex $v_{F}$. This will complete the proof of Theorem 1.0.1. For an abelian group $H$ let $H_{\text {tor }}$ denote its torsion subgroup.

Proposition 11.1.4. There is a set-theoretic inclusion $\Omega_{M \text {,tor }}^{\sigma} \subset \widetilde{K}$ which induces an isomorphism of groups

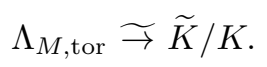

Proof. Clearly, $\Omega_{M \text {,tor }}^{\sigma}$ lies in $M(F)^{1}$ hence in $G(F)^{1}$. Also, every element of $M(F)^{1}$ acts trivially on the apartment $\mathcal{A}_{L}^{\sigma}$, and in particular, fixes $\mathbf{a}_{0}^{\sigma}$. This shows that $\Omega_{M, \text { tor }}^{\sigma} \subset \operatorname{Fix}^{G(F)}\left(v_{F}\right) \cap G(F)^{1}=\widetilde{K}$ (cf. Lemma 8.0.1).

We claim the induced homomorphism $\Omega_{M \text {,tor }}^{\sigma} \rightarrow \widetilde{K} / K$ is an isomorphism. It is injective because

$$
\Omega_{M} \cap K=\Omega_{M} \cap M(F) \cap K=\Omega_{M} \cap M(F)_{1}=\{1\}
$$

(cf. Lemma 4.2.1). 
Let us prove surjectivity. Any coset in $\widetilde{K} / K$ can be represented by an element $x \in \Omega_{M}^{\sigma}$. We need to show this element is torsion. Let $r$ be such that $x^{r} \in K$. But then $x^{r} \in \Omega_{M}^{\sigma} \cap K=\{1\}$ (see above), and we are done.

Corollary 11.1.5. If $M_{L}$ is $L$-split group and $M_{\mathrm{der}}=M_{\mathrm{sc}}$, then $\Lambda_{M}$ is torsionfree, and for every special vertex $v_{F}$, we have $\widetilde{K}_{v_{F}}=K_{v_{F}}$.

Proof. We have

$$
X^{*}(Z(\widehat{M}))_{I}=X^{*}(Z(\widehat{M}))
$$

and the latter is torsion free since $M_{\mathrm{der}}=M_{\mathrm{sc}}$ is equivalent to $Z(\widehat{M})$ being connected.

Remark 11.1.6. The hypotheses on $M$ hold if $G_{\mathrm{der}}=G_{\mathrm{sc}}$ and $G_{L}$ is an $L$-split group.

Corollary 11.1.7. If $G=G_{\mathrm{sc}}$, then $\widetilde{K}=K$ and $\Lambda_{M}$ is torsion-free.

Proof. Observe that since $Z(\widehat{G})=1$ we have $G(F)_{1}=G(F)^{1}=G(F)$. Then use (8.0.1) and 8.0.2).

Of course, this corollary was already known (cf. [BT2, 4.6.32).

11.2. Passing to inner forms. It is of interest to describe $\Lambda_{M}$ explicitly in terms of an appropriate maximal torus $\widehat{T}$ in $\widehat{G}$. For quasi-split groups this has been done in Corollary 11.1.2, (a), which proves that $\Lambda_{M}=X^{*}(\widehat{T})_{I}^{\sigma}=X^{*}\left(\widehat{T}^{I}\right)^{\sigma}$. Here we study the effect of passing to an inner form of a quasi-split group.

Thus, we fix a connected reductive group $G^{*}$ which is quasi-split over $F$. Recall that an inner form of $G^{*}$ is a pair $(G, \Psi)$ consisting of a connected reductive $F$ group $G$ and a $\Gamma$-stable $G_{\text {ad }}^{*}\left(F^{s}\right)$-orbit $\Psi$ of $F^{s}$-isomorphisms $\psi: G \rightarrow G^{*}$. The set of isomorphism classes of inner forms of $G^{*}$ corresponds bijectively to the set $H^{1}\left(F, G_{\mathrm{ad}}^{*}\right)$, by the rule which sends $(G, \Psi)$ to the 1-cocycle $\tau \mapsto \psi \circ \tau(\psi)^{-1}$ for any $\psi \in \Psi$ (cf. Ko97, 5.2).

Now assume $(G, \Psi)$ is an inner form of $G^{*}$. Denote the action of $\tau \in \Gamma$ on $G\left(F^{s}\right)$ (resp. $\left.G^{*}\left(F^{s}\right)\right)$ by $\tau$ (resp. $\left.\tau^{*}\right)$.

Let $A$ be a maximal $F$-split torus in $G$, and let $S$ denote a maximal $F^{\text {un }}$-split torus in $G$ which is defined over $F$ and contains $A$. Such a torus $S$ exists by [BT2, 5.1.12, noting that any $F$-torus which is split over $L$ is already split over $F^{\text {un }}$. Let $T=C_{G}(S)$ and $M=C_{G}(A)$. Then $T$ is a maximal torus of $G$, since the group $G_{F^{u n}}$ is quasi-split. Let $A^{*}, S^{*}, T^{*}$ have the corresponding meaning for the group $G^{*}$, and assume that $T^{*}$ is contained in an $F$-rational Borel subgroup $B^{*}=T^{*} U^{*}$ of $G^{*}$. Of course $T^{*}=C_{G^{*}}\left(A^{*}\right)$ since $G^{*}$ is quasi-split over $F$.

Let $P=M N$ be an $F$-rational parabolic subgroup of $G$ having Levi factor $M$ and unipotent radical $N$. Let $P^{*}$ be the unique standard $F$-rational parabolic subgroup of $G^{*}$ which is $G^{*}\left(F^{s}\right)$-conjugate to $\psi(P)$ for all $\psi \in \Psi$ (cf. Bo, section $3)$. Let $M^{*}$ denote the unique Levi factor of $P^{*}$ which contains $T^{*}$. Let $\Psi_{M}$ denote the set of $\psi \in \Psi$ such that $\psi(P)=P^{*}$ and $\psi(M)=M^{*}$. Then $\Psi_{M}$ is a non-empty $\Gamma$-stable $M_{\mathrm{ad}}^{*}\left(F^{s}\right)$-orbit of $F^{s}$-isomorphisms $M \rightarrow M^{*}$; hence $M$ is an inner form of the $F$-quasi-split group $M^{*}$.

It is clear that $G_{F}$ un and $G_{F \text { un }}^{*}$ are isomorphic, since they are inner forms of each other and are both quasi-split (cf. [Tits, 1.10.3). In fact, it is easy to see that any inner twisting $G_{F^{\text {un }}} \rightarrow G_{F}^{*}$ un over $F^{\text {un }}$ is $G^{*}\left(F^{s}\right)$-conjugate to an isomorphism of 


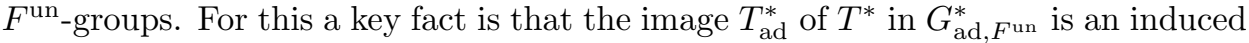
$F^{\text {un }}$-torus. The same remarks obviously apply to $M_{F}$ un and $M_{F}^{*}$ un. Hence we may choose $\psi_{0} \in \Psi_{M}$ such that $\psi_{0}: M \rightarrow M^{*}$ is an $F^{\text {un }}$-isomorphism and $\psi_{0}(S)=S^{*}$ (and thus also $\psi_{0}(T)=T^{*}$ ). Since $\psi_{0}$ restricted to $A$ is defined over $F$, we see that $\psi_{0}(A)$ is an $F$-split subtorus of $T^{*}$ and hence $\psi_{0}(A) \subseteq A^{*}$.

Let $\tilde{\sigma}$ denote any lift in $\Gamma$ of the Frobenius element $\sigma \in \operatorname{Gal}\left(F^{\text {un }} / F\right)$. We may write

$$
\psi_{0} \circ \tilde{\sigma}\left(\psi_{0}\right)^{-1}=\psi_{0} \circ \sigma\left(\psi_{0}\right)^{-1}=\operatorname{Int}\left(m_{\sigma}^{*}\right)
$$

for an element $m_{\sigma}^{*} \in N_{M^{*}}\left(S^{*}\right)\left(F^{s}\right)$ whose image in $M_{\mathrm{ad}}^{*}\left(F^{s}\right)$ is well defined. As operators on $X_{*}\left(T^{*}\right)=X^{*}\left(\widehat{T^{*}}\right)$, we may write

$$
\psi_{0} \circ \sigma\left(\psi_{0}\right)^{-1}=w_{\sigma}^{*}
$$

for a well-defined element $w_{\sigma}^{*} \in W\left(M^{*}, S^{*}\right)\left(F^{\text {un }}\right)$. Denote by $w_{\sigma}$ the preimage under the isomorphism $\psi_{0}: W(M, S)\left(F^{\mathrm{un}}\right) \widetilde{\rightrightarrows} W\left(M^{*}, S^{*}\right)\left(F^{\mathrm{un}}\right)$ of $w_{\sigma}^{*}$. Then (11.2.1) translates into the equality

$$
\sigma \circ \psi_{0}^{-1} \circ\left(\sigma^{*}\right)^{-1} \circ \psi_{0}=w_{\sigma}
$$

of operators on $X_{*}(T)=X^{*}(\widehat{T})$. In defining $w_{\sigma} \in W(M, S)$, we fixed the objects $A$ and $S$ (needed to specify the ambient group $W(M, S)$ ) and along the way we also chose several additional objects: $P, A^{*}, S^{*}, B^{*}$, and an element $\psi_{0} \in \Psi_{M}$ such that $\psi_{0}(S)=S^{*}$ and $\psi_{0}: M \rightarrow M^{*}$ is $F^{u n}$-rational. It is straightforward to check that the element $w_{\sigma} \in W(M, S)$ is independent of all of these additional choices.

11.3. Inner forms of split groups. In this subsection we assume $G^{*}$ is $F$-split. Then $A^{*}=S^{*}=T^{*}$, and $G_{F^{u n}}$ and $M_{F \text { un }}$ are split groups. In particular, the relative Weyl group $W\left(M^{*}, S^{*}\right)$ coincides with the absolute Weyl group $W\left(M^{*}, T^{*}\right)$. Using $\psi_{0}$ as above, we may regard $w_{\sigma}$ as an element of $W(M, S)=W(M, T)^{I}=$ $W(\widehat{M}, \widehat{T})^{I}$.

For the next lemma, we need to recall the notion of cuspidal elements of Weyl groups. Let $(W, S)$ be any Coxeter group with a finite set $S$ of simple reflections. We say $w \in W$ is cuspidal if every conjugate of $w$ is elliptic, that is, every conjugate $w^{\prime}$ has the property that any reduced expression for $w^{\prime}$ contains every element of $S$. Note that the identity element of $W$ is cuspidal if and only if $S=\emptyset$, in which case $W$ itself is trivial.

Lemma 11.3.1. (a) The element $w_{\sigma}$ is a cuspidal element of the absolute Weyl group $W(M, T)$ of $M$.

(b) The group $M$ is of type $A$ and the element $w_{\sigma}$ is a Coxeter element of $W(M, T)$.

(c) We have the equality $Z(\widehat{M})=\widehat{T}^{w_{\sigma}}$.

Proof. Part (a). We may assume $M \neq T$ and hence $W(M, T)$ is not trivial. Suppose the assertion is false. Then there is a notion of simple positive root for $M, T$ and a corresponding Coxeter group structure on $W(M, T)$, for which $w_{\sigma}$ is not an elliptic element. Let $s_{i}$ denote a simple reflection in $W(M, T)$ which does not appear in a reduced expression for $w_{\sigma}$. Then the corresponding fundamental coweight $\lambda_{i} \in X_{*}(T / Z(M))$ for $M_{\text {ad }}$ is fixed by $w_{\sigma}$. It is also fixed by $\psi_{0}^{-1} \circ\left(\sigma^{*}\right)^{-1} \circ \psi_{0}$. Thus by (11.2.2) $\lambda_{i}$ is fixed by $\sigma$, and $\lambda_{i}\left(\mathbb{G}_{m}\right)$ is an $F$-split torus in $M_{\text {ad }}$. This contradicts the fact that $M_{\text {ad }}$ is anisotropic over $F$. 
Part (b). Since every anisotropic F-group is type A (cf. Kneser $[\mathrm{Kn}$ and BruhatTits [BT3] 4.3), the group $M$ is type $A$. For type A groups, every cuspidal element in the Weyl group is Coxeter, as may be seen using cycle decompositions of permutations. Thus, the cuspidal element $w_{\sigma}$ is a Coxeter element of $W(M, T)$.

Part (c). It is enough to prove the following statement: if $\mathcal{G}$ is a type A connected reductive complex group with maximal torus $\mathcal{T}$, and if $w \in W(\mathcal{G}, \mathcal{T})$ is a Coxeter element, then $Z(\mathcal{G})=\mathcal{T}^{w}$. First, if $\mathcal{G}=\mathrm{PGL}_{n}$, a simple computation shows that $\mathcal{T}^{w}=1=Z(\mathcal{G})$. Since $\mathcal{G}_{\text {ad }}$ is a product of projective linear groups and $w$ corresponds to a product of Coxeter elements, this also handles the case of adjoint groups. In the general case, note that an element $t \in \mathcal{T}^{w}$ maps to $\left(\mathcal{T}_{\text {ad }}\right)^{w}=1$ in $\mathcal{G}_{\text {ad }}$, hence $t \in \operatorname{ker}\left(\mathcal{G} \rightarrow \mathcal{G}_{\text {ad }}\right)=Z(\mathcal{G})$.

Corollary 11.3.2. If $G$ is an inner form of an $F$-split group, then

$$
\Lambda_{M}=X^{*}(Z(\widehat{M}))=X^{*}\left(\widehat{T}^{\sigma}\right)=X_{*}(T)_{\sigma} .
$$

Proof. The element $\sigma^{*}$ acts trivially on $Z(\widehat{M}) \hookrightarrow \widehat{T}^{*}$, since $T^{*}$ is $F$-split. Moreover, $w_{\sigma} \in W(M, T)$ acts trivially on $X^{*}(Z(\widehat{M}))$. Then using (11.2.2) it follows that $\sigma$ acts trivially on $X^{*}(Z(\widehat{M}))_{I}=X^{*}(Z(\widehat{M}))$. This proves the first equality.

The second equality follows similarly using Lemma 11.3.1, (c), and the third equality is apparent.

\section{The TRANSFER HOMOMORPHISM}

Now we return to the conventions and notation of subsection 11.2, Let $\mathcal{A}_{L}^{S}$ (resp. $\mathcal{A}_{L}^{S^{*}}$ ) denote the apartment of $\mathcal{B}(G(L))$ (resp. $\mathcal{B}\left(G^{*}(L)\right)$ ) corresponding to $S$ (resp. $\left.S^{*}\right)$. The twisting $\psi_{0}$ gives an isomorphism $X_{*}(S)_{\mathbb{R}} \rightarrow X_{*}\left(S^{*}\right)_{\mathbb{R}}$ of the real vector spaces underlying these apartments. Let $K$ (resp. $K^{*}$ ) denote a special maximal parahoric subgroup of $G(F)$ (resp. $G^{*}(F)$ ) corresponding to a special vertex in $\left(\mathcal{A}_{L}^{S}\right)^{\sigma}$ (resp. $\left.\left(\mathcal{A}_{L}^{S^{*}}\right)^{\sigma^{*}}\right)$. Then our goal is to define a canonical algebra homomorphism

$$
t: \mathcal{H}_{K^{*}}\left(G^{*}\right) \rightarrow \mathcal{H}_{K}(G)
$$

We expect $t$ will play a role in the study of Shimura varieties with parahoric level structure and in some related problems in $p$-adic harmonic analysis. These issues will be addressed on another occasion.

\subsection{Relating the relative Weyl groups for $G^{*}$ and $G$.}

Proposition 12.1.1. Any twist $\psi_{0} \in \Psi_{M}$ induces a map

$$
W(G, A) \rightarrow W\left(G^{*}, A^{*}\right) / W\left(M^{*}, A^{*}\right) .
$$

Proof. For $w \in W(G, A)$, choose a lift $n \in N_{G}(S)^{\sigma}$ (cf. Lemma 5.0.1). Write

$$
\sigma \circ \psi_{0}^{-1} \circ\left(\sigma^{*}\right)^{-1} \circ \psi_{0}=\operatorname{Int}\left(m_{\sigma}\right)
$$

for an element $m_{\sigma} \in N_{M}(S)\left(F^{s}\right)$. Set $m_{*}=\psi_{0}\left(\sigma^{-1}\left(m_{\sigma}\right)\right) \in N_{M^{*}}\left(S^{*}\right)\left(F^{s}\right)$. Using $\sigma(n)=n$ and the fact that $\psi_{0}(n)$ normalizes $M^{*}$, we obtain

$$
\begin{aligned}
\left(\sigma^{*}\right)^{-1}\left(\psi_{0}(n)\right) & =m_{*} \psi_{0}(n) m_{*}^{-1} \\
& =\psi_{0}(n) \cdot\left(\psi_{0}(n)^{-1} m_{*} \psi_{0}(n) m_{*}^{-1}\right) \\
& \in \psi_{0}(n) N_{M^{*}}\left(S^{*}\right) .
\end{aligned}
$$


Thus $n \mapsto \psi_{0}(n)$ induces a well-defined map

$$
W(G, A) \rightarrow\left(W\left(G^{*}, S^{*}\right) / W\left(M^{*}, S^{*}\right)\right)^{\sigma^{*}}
$$

The natural map $W\left(G^{*}, S^{*}\right)^{\sigma^{*}} \rightarrow\left(W\left(G^{*}, S^{*}\right) / W\left(M^{*}, S^{*}\right)\right)^{\sigma^{*}}$ is surjective. Indeed, the choice of an $F$-rational Borel subgroup of $G^{*}$ containing $T^{*}$ gives us a notion of length on $W\left(G^{*}, S^{*}\right)$ which is preserved by $\sigma^{*}$, so that the minimallength representatives of $\sigma^{*}$-fixed cosets in $W\left(G^{*}, S^{*}\right) / W\left(M^{*}, S^{*}\right)$ are fixed by $\sigma^{*}$. It follows that

$$
W\left(G^{*}, S^{*}\right)^{\sigma^{*}} / W\left(M^{*}, S^{*}\right)^{\sigma^{*}}=\left(W\left(G^{*}, S^{*}\right) / W\left(M^{*}, S^{*}\right)\right)^{\sigma^{*}}
$$

Thus, we have a well-defined map

$$
W(G, A) \rightarrow W\left(G^{*}, S^{*}\right)^{\sigma^{*}} / W\left(M^{*}, S^{*}\right)^{\sigma^{*}}=W\left(G^{*}, A^{*}\right) / W\left(M^{*}, A^{*}\right)
$$

(cf. Remark 6.1.3).

12.2. Definition of $t: \mathcal{H}_{K^{*}}\left(G^{*}\right) \rightarrow \mathcal{H}_{K}(G)$. The isomorphism

$$
\widehat{\psi_{0}}: Z\left(\widehat{M^{*}}\right) \stackrel{\Im}{\rightarrow}(\widehat{M})
$$

is Galois-equivariant. Combined with the canonical inclusion $Z\left(\widehat{M^{*}}\right) \hookrightarrow \widehat{T^{*}}$ we see that $\widehat{\psi_{0}}$ induces a homomorphism

$$
\psi_{0}: X^{*}\left(\widehat{T^{*}}\right)_{I}^{\sigma^{*}} \rightarrow X^{*}(Z(\widehat{M}))_{I}^{\sigma}
$$

Since $W\left(M^{*}, A^{*}\right)$ induces the trivial action on $Z\left(\widehat{M^{*}}\right)$, it follows using Proposition 12.1.1 that (12.2.1) is equivariant with respect to the map $W(G, A) \rightarrow$ $W\left(G^{*}, A^{*}\right) / W\left(M^{*}, A^{*}\right)$, in an obvious sense. We thus get an algebra homomorphism

$$
\psi_{0}: \mathbb{C}\left[X^{*}\left(\widehat{T^{*}}\right)_{I}^{\sigma^{*}}\right]^{W\left(G^{*}, A^{*}\right)} \rightarrow \mathbb{C}\left[X^{*}(Z(\widehat{M}))_{I}^{\sigma}\right]^{W(G, A)}
$$

Since $\Psi_{M}$ is a torsor for $M_{\mathrm{ad}}^{*}$, one can check that this homomorphism is independent of the choice of $\psi_{0}$ in $\Psi_{M}$. In fact, it depends only on the choice of $A$ and $A^{*}$. Therefore, it makes sense to denote it by $t_{A^{*}, A}$ in what follows. It is easy to check that this homomorphism is surjective when $G^{*}$ is split over $F$.

Definition 12.2.1. Fix $A$ and $A^{*}$ as above. Define $t: \mathcal{H}_{K^{*}}\left(G^{*}\right) \rightarrow \mathcal{H}_{K}(G)$ to be the unique homomorphism making the following diagram commute

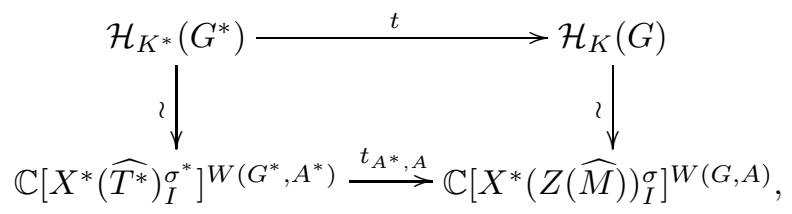

where the vertical arrows are the Satake isomorphisms.

Obviously, $t$ depends on $K$ and $K^{*}$. It is easy to see that $t$ is independent of all other choices used in its construction. Also, if $G^{*}$ is split over $F, t$ is surjective. 
12.3. Compatibilities with constant term homomorphisms. Let $A, A^{*}, K$, and $K^{*}$ be fixed as above. Let $H$ be a semi-standard $F$-Levi subgroup of $G$; this means that $H=C_{G}\left(A_{H}\right)$ for some subtorus $A_{H} \subseteq A$. Let $H^{*}$ be a semi-standard $F$-Levi subgroup of $G^{*}$, so that $H^{*}=C_{G^{*}}\left(A_{H^{*}}^{*}\right)$ for a subtorus $A_{H^{*}}^{*} \subset A^{*}$. We have $M \subseteq H$ and $T^{*} \subseteq H^{*}$. Let us suppose that some inner twist $G \rightarrow G^{*}$ restricts to give an inner twist $H \rightarrow H^{*}$.

For example, for any $\psi_{0} \in \Psi_{M}$ as above, we could take $A_{H}$ to be any subtorus of $A$ and set $A_{H^{*}}^{*}=\psi_{0}\left(A_{H}\right)$ (recalling that $\psi_{0}(A) \subseteq A^{*}$ ).

Choose any $F$-rational parabolic subgroup $P_{H}=H N_{H}$ of $G$ with unipotent radical $N_{H}$ which contains $H$ as a Levi factor. Recall the constant term map $c_{H}^{G}: \mathcal{H}_{K}(G) \rightarrow \mathcal{H}_{H \cap K}(H)$, which is defined by

$$
c_{H}^{G}(f)(h)=\delta_{P_{H}}^{1 / 2}(h) \int_{N_{H}(F)} f(h n) d n,
$$

for $h \in H(F)$, where the Haar measure $d n$ on $N_{H}(F)$ gives $N_{H}(F) \cap K$ measure 1 . We have a commutative diagram

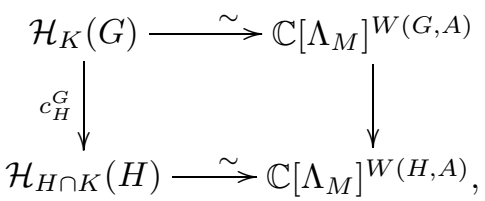

where the horizontal arrows are the Satake isomorphisms, and the right vertical arrow is the inclusion homomorphism. It follows that $c_{H}^{G}$ is an injective algebra homomorphism which is independent of the choice of $F$-rational parabolic subgroup $P_{H} \subseteq G$ which contains $H$ as a Levi factor.

The following proposition is proved using (12.3.2) and the definitions.

Proposition 12.3.1. The following diagram commutes:

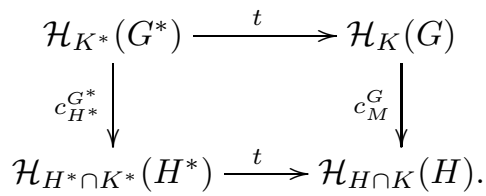

Taking $H=M$, the diagram shows that in order to compute $t$, it is enough to compute it in the case where $G_{\text {ad }}$ is anisotropic. In that case, if $f \in \mathcal{H}_{K^{*}}\left(G^{*}\right)$, the function $t(f)$ is given by summing $f$ over the fibers of the Kottwitz homomorphism $k_{G^{*}}(F)$.

\section{REFERENCES}

[Bo] A. Borel, Automorphic L-functions, In: Automorphic Forms, Representations and Lfunctions, Proc. Sympos. Pure Math., vol. 33, part 2, Amer. Math. Soc., Providence, RI, 1979, pp. 27-61. MR 546608 (81m:10056)

[Bou] N. Bourbaki, Groupes et Algèbres de Lie, Chap. 4, 5, et 6, Masson, 1981. MR647314 (83g:17001)

[BT1] F. Bruhat and J. Tits, Groupes réductifs sur un corps local. I, Inst. Hautes Études Sci. Publ. Math. 41 (1972), 5-251. MR0327923 (48:6265)

[BT2] F. Bruhat and J. Tits, Groupes réductifs sur un corps local. II, Inst. Hautes Études Sci. Publ. Math. 60 (1984), 5-184. 
[BT3] F. Bruhat and J. Tits, Groupes réductifs sur un corps local. III, Journal Fac. Science University Tokyo 34 (1987), 671-698. MR.927605 (89b:20099)

[Car] P. Cartier, Representations of p-adic groups: a survey In: Automorphic Forms, Representations and L-functions, Proc. Sympos. Pure Math., vol. 33, part 1, Amer. Math. Soc., Providence, RI, 1979, pp. 111-155. MR546593 (81e:22029)

[H05] T. Haines, Introduction to Shimura varieties with bad reduction of parahoric type, Clay Math. Proc. 4, (2005), 583-642. MR2192017 (2006m:11085)

[H09] T. Haines, The base change fundamental lemma for central elements in parahoric Hecke algebras, Duke Math. J. 149, no. 3 (2009), 569-643. MR2553880

[HKP] T. Haines, R. Kottwitz, A. Prasad, Iwahori-Hecke algebras, math.RT/0309168. Preprint.

[HR] T. Haines, M. Rapoport, Appendix: On parahoric subgroups, Advances in Math. 219 (1), (2008), 188-198; appendix to: G. Pappas, M. Rapoport, Twisted loop groups and their affine flag varieties, Advances in Math. 219 (1), (2008), 118-198. MR2435422 (2009g:22039)

[Kn] M. Kneser, Galois-Kohomologie halbeinfacher algebraischer Gruppen über p-adischen Körpern. II. Math. Zeitschr. 89, 250-272 (1965). MR0188219(32:5658)

[Ko97] R. Kottwitz, Isocrystals with additional structure. II, Compositio Math. 109, 255-339 (1997). MR1485921 (99e:20061)

[Kr] N. Krämer, Local models for ramified unitary groups, Abh. Math. Sem. Univ. Hamburg 73 (2003), 67-80. MR 2028507 (2004k:14037)

[Land] E. Landvogt, A compactification of the Bruhat-Tits building, Lecture Notes in Mathematics 1619, Springer, 1996, 152 pp. + vii. MR1441308 (98h:20081)

[PR] G. Pappas, M. Rapoport, Local models in the ramified case. III. Unitary groups., J. Inst. Math. Jussieu 8 (2009), no. 3, 507-564. MR2516305

[Rap] M. Rapoport: A guide to the reduction modulo $p$ of Shimura varieties. Astérisque 298 (2005), 271-318. MR2141705 (2006c:11071)

[Tits] J. Tits, Reductive groups over local fields, Proc. Symp. Pure. Math. 33 (1979), part. 1, pp. 29-69. MR.546588 (80h:20064)

University of Maryland, Department of Mathematics, College Park, Maryland $20742-4015$

E-mail address: tjh@math.umd.edu

University of Maryland, Department of Mathematics, College Park, Maryland $20742-4015$

E-mail address: srostami@math.umd.edu 